\title{
Optimization Study On The Location Of An Active Chilled Beam: Effect On Thermal Comfort And Energy Consumption
}

\author{
Nikhilesh Ghanta ${ }^{1}$, Barry Coflan ${ }^{2}$, Leon Glicksman ${ }^{1}$ \\ ${ }^{1}$ Massachusetts Institute of Technology, Cambridge, USA \\ ${ }^{2}$ Schneider Electric, Andover, USA
}

\begin{abstract}
The market for chilled beams is predicted to grow by more than $150 \%$ in the next 10 years and the primary reason for their increasing popularity is their low operating costs and high energy savings potential. It becomes imperative that the design and installation of such a system are optimized for maximum energy savings. In the present work, we study the influence of the location of the active chilled beam in a room on the thermal comfort of the occupants and the energy usage for attaining that thermal comfort. When located optimally in the room, the chilled beam can counter the impact of adverse outdoor environments while simultaneously maintaining optimal energy usage. Computational Fluid Dynamics (CFD) simulations are employed to model the room in consideration and simulate during the summer months, with experiments performed on one orientation of the room and the chilled beam for the CFD validation. Thermal comfort is quantized using the Fanger's Predictive Mean Vote (PMV) model. Optimal location calculated using Kriging modelling and Genetic algorithm places the chilled beam at three-fourths mark $(\mathrm{x} / \mathrm{L}=0.75-0.8)$ along the length of the room closer to the external window.
\end{abstract}

\section{Introduction}

Chilled beams are considered an efficient alternate to conventional fan-coil air conditioning systems. Chilled beams typically have no moving parts, utilize minimum fan energy and have a better air distribution system, leading to higher energy efficiency and better thermal comfort. These perks are contributing to their increased demand across the world, especially in commercial spaces. Warm air rises, goes through the chilled beam, gets cooled by the interaction with chilled recirculated water and falls back into the occupied space. In active chilled beams, the ventilation air is directly delivered to the beam and is integrated to the air-handling system, while a passive chilled beam needs to have a special ventilation system to ensure proper air quality. Chilled beams utilize the Coanda effect to help disperse the conditioned air throughout the room, according to the design settings. They create an induction for the warm air to automatically rise faster and pass through the chilled beam, thereby reducing the need for a fan coil to drive air flow. However, they typically have higher initial costs and can lead to condensation if not properly designed. Another major drawback is the lack of proper design criteria for positioning of a chilled beam in a room. This present work aims to address this issue, by identifying the optimal location of a chilled beam in a general commercial conference room, consisting of an external window.

Numerous studies have been conducted to understand the operation of active and passive chilled beams and their impact on the comfort levels of humans in the occupied zones. The air flow pattern determines how quickly the conditioning happens in the room (Muller et al, 2004) and the external weather conditions play a major role in this interaction. Air flow in rooms is primarily driven by two factors- air conditioning and buoyancy flows from heat sources. These flow patterns and interactions were studied by Koskela et al. (2012), for the case of space cooling. More recent studies on chilled beams have been performed by Kim et al. (2018) and Wu et al. (2018).

The current study involves an active chilled beam with a 4-pipe connection, located in a commercial conference room in Andover, Massachusetts (USA). This manuscript is based on experiments conducted in the room and computational simulations performed using ANSYS Fluent and Airpak. Commercial spaces and conference rooms typically aren't occupied at all times, but periodically and frequently. Also, chilled beams are known to perform better for spaces where the air-conditioning system is sized based on cooling and heating loads, rather than the ventilation. 
Periodically and intermittently occupied commercial spaces fall into this category. Buildings with heat gain from equipment and solar radiation are also good avenues for installation of chilled beams. The present work also deals with such a case- with internal heat gains and a large external window contributing to a considerable amount of solar heat gains. The primary objective of this study is to understand the effectiveness of chilled beams especially in extreme external weather conditions in simultaneously achieving thermal comfort and consuming the least energy possible.

\section{Methodology}

The conference room has floor dimensions of $5.8 \mathrm{~m} \mathrm{x}$ $5.5 \mathrm{~m}$ and has a ceiling-floor distance of $2.8 \mathrm{~m}$. It has one side of the longer dimension as an external wall, facing North. The external wall has a large window, completely covering the entire wall area from a height of $0.75 \mathrm{~m}$ until the ceiling. The other three walls are internal walls connected to other rooms of the building. The room has a false ceiling alongside the chilled beam ducts, rendering almost zero heat transfer through the top boundary of the room. The floor is concrete-based and acts as a thermal mass. The chilled beam is located $1 \mathrm{~m}$ away from the external window and parallel to it and is $2.8 \mathrm{~m}$ in length. The wall opposite the external window has a door with a leakage area around it. The door is $0.8 \mathrm{~m}$ wide and 2.5 $\mathrm{m}$ tall. The room has a projector connected to the ceiling and a couple of tables with multiple chairs, providing occupancy for a maximum of 8 people to sit in. Apart from the incoming solar radiation through the external window, the other sources of heat generation are the projector, humans, and laptops. Lighting used in the room is almost zero due to the large unshaded external window. Depending on the temperature setpoint in the thermostat located on the wall opposite the external window, the chilled beam either cools or heats the space.

Experiments were conducted in the months of May, during which the outdoor temperature in Andover, Massachusetts was in the range of $20-25^{\circ} \mathrm{C}$. The measurements of velocity were conducted using hotwire anemometers (TSI VelociCalc Air Velocity Meter 8345; +-0.015m/s accurate). Temperature readings at various locations in the interior room space were recorded using multiple thermocouples (TSI VelociCalc $8345 ; \quad 0.3^{\circ} \mathrm{C}$ accurate) and surface temperatures were measured using an Infrared Camera (Flir E5 Wi-Fi Infrared Camera; +-2\% accurate. Additionally, the concentration of carbon dioxide in the room was also monitored (Telaire $7001 \mathrm{CO} 2$ sensor; +-50 ppm accurate). This is crucial in the operation of the active chilled beam with control logic to bring in the outdoor fresh air to meet a specified set point of $\mathrm{CO}_{2}$ concentration. This in effect would add additional conditioning load to the chilled beam.

Computational Fluid Dynamics (CFD) was implemented through the commercial softwares of ANSYS and Airpak. The test room was modelled to the actual dimensions and all the features of the room included, as shown in Figure 1. Radiation was modelled using surface-to-surface radiation calculations. K-epsilon RNG model was implemented to model turbulence characteristics in the room. The chilled beams were modelled to deliver a constant flow rate of air, according to the manufacturer specifications. For the purpose of the study, the fan coil unit integrated with the chilled beam was assumed to be operating at constant air flow rates. The rate of flow of chilled water in the beam was also assumed to be constant.

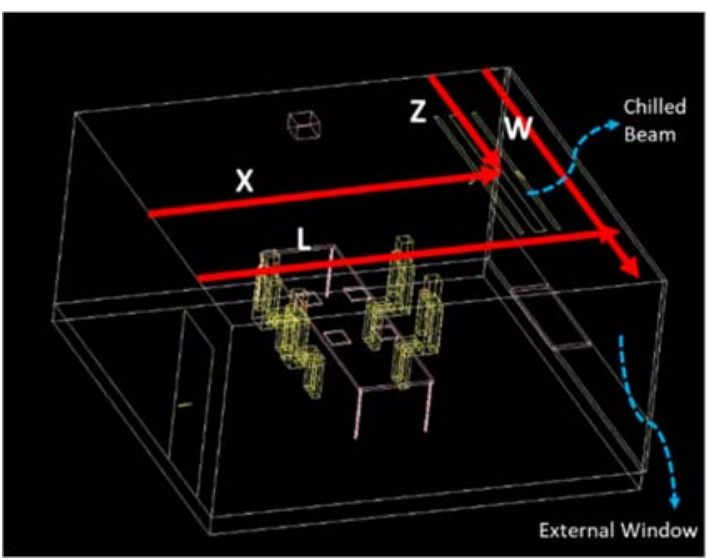

Figure 1: 3D model of the experimental room, as modelled in Airpak, indicating the variables used in this study.

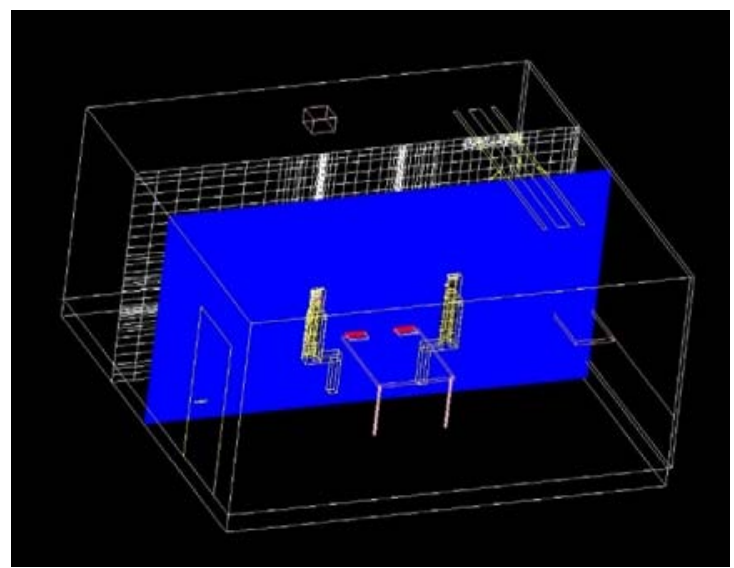

Figure 2: Plane cutting across the humans in the middle of the room, on which the CFD results are realized and presented in later sections of the paper 


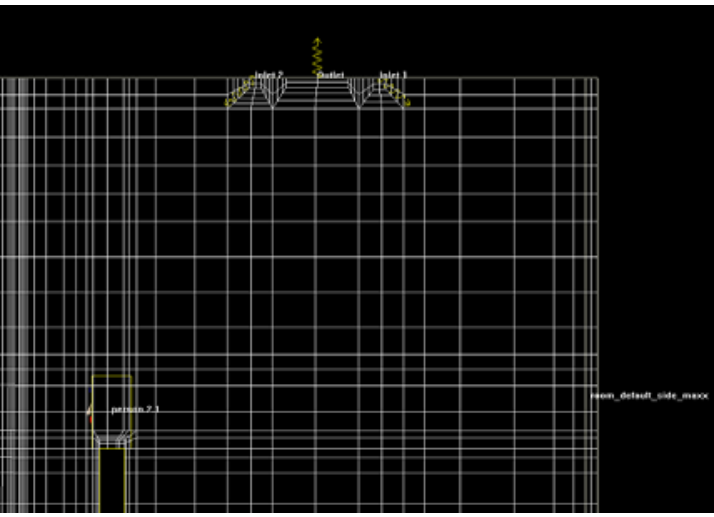

Figure 3: Dynamic meshing adopted for the CFD simulations, shown near the chilled beam system in the room

Weather data files from the Boston weather station were used for simulating the outdoor conditions. The epw files were provided as inputs to the model, with the external temperature, relative humidity and solar loading as the primary input features. The heat sources in the room were modelled as solid objects with specific heat emission rates. These include human beings, electronic equipment like laptops and projectors and lighting. Three of the four boundaries of the room were modelled as adiabatic surfaces, with the fourth one being the external window. The corridor connecting the door with the other part of the building was assumed to be maintained at a relatively uniform temperature and relative humidity. This corridor plays a role in incorporating the end effects of flow through the door of the room and is hence important to be considered for modelling. The ceiling is considered adiabatic, with a false ceiling in place for the installation of a chilled beam. The floor is modelled as a concrete slab of finite thickness to account for its thermal mass properties. The simulations were performed considering 4 occupants. The basic governing equations involved in the simulations using the finite volume method are the continuity equations and the conservation of momentum and energy. The actual compressible version of these equations used is shown below.

$$
\begin{gathered}
\frac{\partial \rho}{\partial t}+\nabla \cdot(\rho U)=0 \\
\frac{\partial(\rho U)}{\partial t}+\nabla \cdot(U \cdot \rho U)-\rho g=-\nabla p+ \\
\nabla \cdot\left[\mu\left(\nabla U+(\nabla U)^{T}\right)\right] \\
\frac{\partial \rho c T}{\partial t}+\nabla \cdot(\rho c U T)=\nabla \cdot(k \nabla T)+P \nabla \cdot V
\end{gathered}
$$

Mesh generation is dynamically performed as illustrated in Figure 3, with finer mesh sizes near the surface of solid objects and courser sizes away from them. The minimum size of the mesh was determined by the minimum boundary layer thickness against any particular surface, in order to capture the boundary physics in the model. Grid independence study was done to determine the optimum number of mesh points in the model, as shown in Figure 4. A nominal value of mesh size was chosen where the change in accuracy of the simulated values of temperature and velocity were found to be less than $1 \%$ when doubling the number of grid points.

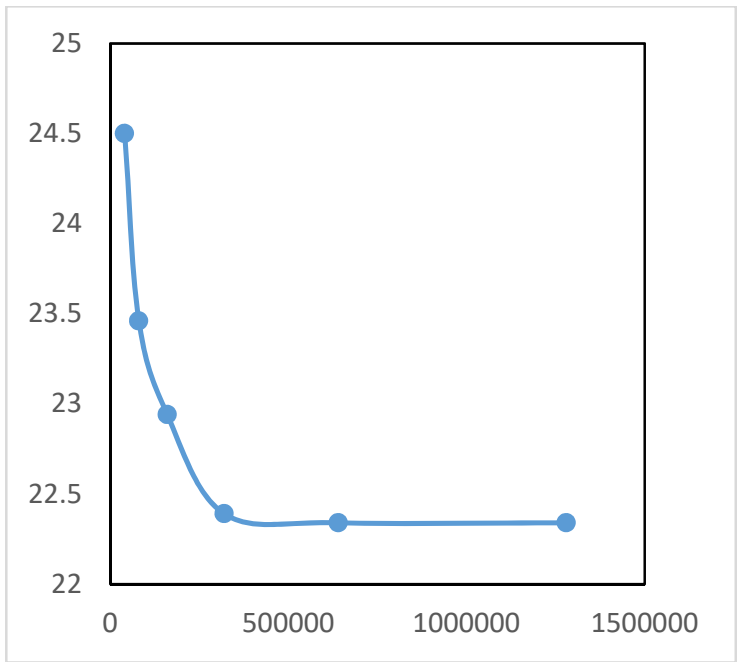

Figure 4: Grid Independence Study for CFD analysis to determine the optimal number of grid points for meshing. The variable in testing was air temperature $\left(\right.$ in ${ }^{\circ} \mathrm{C}$ ) at a specific location in the room, indicated on the $y$-axis.

One of the most widely used thermal comfort models for mechanical conditioning scenarios is the Fanger's PMV (Predictive Mean Vote) model. Fanger's PMV correlation is based on the identification of sweating rate and skin temperature needed for the optimal thermal conditions. It is defined by six different variables- air temperature, relative air velocity, mean radiant temperature, mean aid humidity, clothing insulation and metabolic rate. One set of equations to determine them are shown below, extracted from the data of Rohles and Nevins (1971).

$$
\begin{aligned}
& \mathrm{T}_{\text {sk, req }}=96.3-0.156^{*} \mathrm{q}_{\text {met,heat }} \\
& \mathrm{q}_{\text {sweat, req }}=0.42 *\left(\mathrm{q}_{\text {met, heat }}-18.43\right)
\end{aligned}
$$

PMV is calculated using the below set of equations. 
$\mathrm{PMV}=4+(0.303 \exp (-0.036 \mathrm{H})+0.0275) \times\{6.57+$ $0.46 \mathrm{H}+0.31 \mathrm{~Pa}+0.0017 \mathrm{H}^{*} \mathrm{~Pa}+0.0014 \mathrm{H}^{*} \mathrm{Ta}-4.13$ fcl $(1+0.01 \mathrm{dT})(\mathrm{Tcl}-\mathrm{Tr})-\mathrm{hc} * \mathrm{fcl}(\mathrm{Tcl}-\mathrm{Ta})\}$

Where each individual variable term has its own separate equation based on the surrounding conditions of temperature, humidity, pressure etc. PMV is measured on a scale of -3 to 3 , positive numbers indicating hot conditions and negative indicating cold. A higher magnitude of the PMV value indicates that the condition is more uncomfortable. Ideally, a range of +0.75 to -0.75 is considered a well-conditioned space for humans to occupy. PMV has been known to be challenging to use in the real world, in terms of its dependency on physiological conditions and subjective perception of occupants. However, it is one of the best known and accurate thermal comfort model available for mechanically air-conditioned buildings. The next better and widely used alternative to PMV is the Adaptive Thermal Comfort standard, which is valid only for buildings without mechanical airconditioning.

The variables of interest in this work are the relative positioning of the chilled beams, with respect to the room dimensions. They are treated as non-dimensional numbers, considering ratios of $\mathrm{x}$ to $\mathrm{L}$ and $\mathrm{z}$ to $\mathrm{W}$, as indicated in Figure 1. The variables $\mathrm{x} / \mathrm{L}$ and $\mathrm{w} / \mathrm{D}$ vary from 0 to 1 , with both values zero indicating top left corner and 1 indicating bottom right corner as represented in Figure 1. The objective functions are taken to be the mean PMV values of the occupancy space in the room and the operational time of the air conditioning system, represented by the transient time the model takes to achieve the set-back temperature in the space right next to the thermostat. In the current study, the thermostat setpoint is taken as $23^{\circ} \mathrm{C}$ and the operational time is defined as the time taken for the computational gridpoint near the physical location of the thermostat to attain a temperature of $23^{\circ} \mathrm{C}$. The thermostat is generally located on the wall opposite the external window, on the other side of the positioning of the door. In calculating mean PMV, the central region of the room is considered as primary occupancy space. Owing to different normalizations of both these objective functions, weighted means method of unifying into a single objective function will not prove effective. It becomes imperative to solve this case as a multi-objective optimization problem. Additionally, the simulations performed in this study are limited to the summer months, i.e., mechanical cooling of the occupied spaces. A similar analysis can also be extended to winter seasons.

A preliminary validation study was performed with the experimental readings of temperature and velocity inside multiple locations of the room. The vertical gradient of temperature was also checked for in the accuracy of the numerical simulations. Figures 5 and 6 compare the temperature and velocity readings from experiments with CFD simulations adjacent to the door, with the door being kept open. The CFD simulations gave sufficiently accurate values and trends of temperature and velocities as compared to the experiments in the test room, keeping the error percentage around 8 .

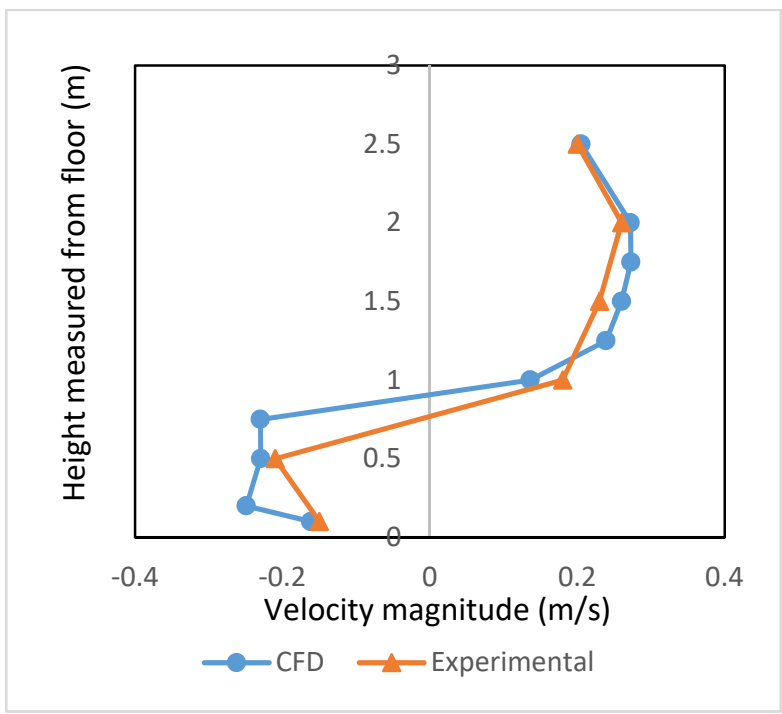

Figure 5: Validation of the CFD studies with experimental data-air velocity at various vertical points inside the room adjacent to the door, with door open

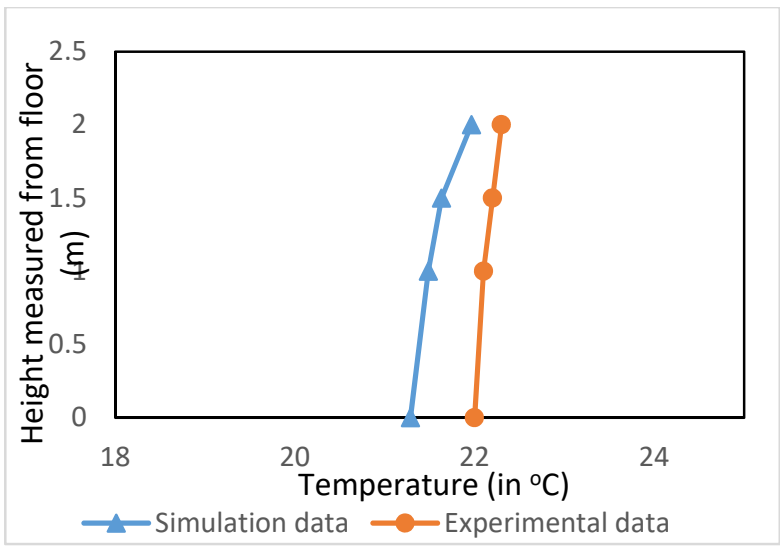

Figure 6: Validation of the CFD studies with experimental data-air temperature at various vertical points inside the room adjacent to the door, with door open 


\section{Modelling and Optimization of CFD results}

CFD is a very comprehensive, yet computationally intensive and time consuming modelling technique. It is logistically not possible to run a CFD simulation every time any optimization algorithm needs a data point. This is usually dealt by employing a simpler modelling technique to fit the particular results of a CFD simulation that we are looking for- in this case, the mean PMV values and operational time of the air conditioning equipment. A detailed hierarchical process flow is illustrated in Figure 7.

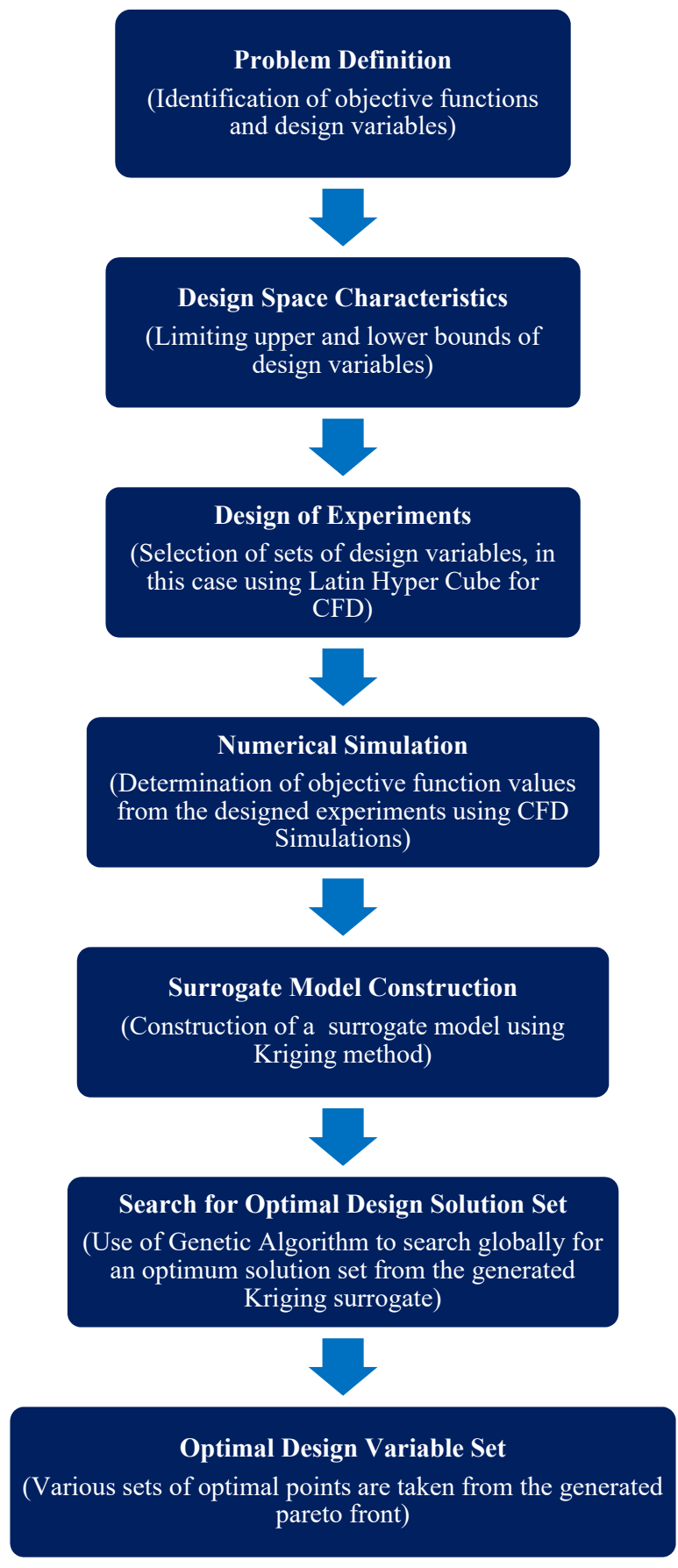

Figure 7: Flow chart detailing the modelling and optimization procedure adopted in this study

In order to generate the sample set of input points to run the CFD simulations, Latin Hyper Cube Sampling is implemented. Ten sampling points were created using LHS. Owing to the relatively simple dependence of the objective functions on the variables, ten simulations were good enough to get a good model fit. However, as one tries to expand this work to more 
complex parameters (varying internal heat sources, varying chilled beam outlet parameters etc.) and cases (example- simultaneous simulation of summer and winter month scenarios), a larger number of sample points are recommended- at least of the order of full factorial sampling. While implementing LHS in generating the ten points, the orthogonality feature was invoked, ensuring an even better distribution of the sampling points in the domain space. With the orthogonality incorporated into LHS, the total sample space is divided into equally probable subspaces. All the sample points are chosen simultaneously such that the total ensemble of chosen sample points represents a Latin Hyper-cube sample and each subspace is sampled with the same density. This way, LHS produces a better sampling data from the domain of the room while being dimension-independent.

Kriging is a method of interpolation for which the interpolated values are modelled by a Gaussian process. It has sufficient flexibility to represent multimodal and non-linear functions, even for a small set of data points. This modelling process is governed by prior covariances. DACE (Design and Analysis of Computer Experiments) toolbox in MATLAB R2018b was used to model using the Kriging method, which was developed by Lophaven et al. (2002). Required changes are made to fit the toolbox to the present study.

The optimizer used in the present study to locate the optimum points from the models developed by algorithms discussed above is the Genetic Algorithm (GA). The preliminary advantage of GA is its ability to converge to the global optimum. Hence, any level of multi-modality can be easily taken care of by GA. Another advantage offered by GA is its usage of just the objective function values in its functional working, and not the derivative of the objective functions involved. This makes it a derivative-free global optimizer, hence allowing it to be robust in its application. GA is coded in MATLAB R2018b and was integrated with the modelling algorithms. Different parameters in GA like the size of the initial population, generation number, cross-over, mutation and convergence are adjusted to suit the present problem. As the chilled beam is moved farther from the external window, the PMV is observed to increase, but the operational time decreases. However, we want an ideal case of lower PMV values and lower operational time, making the two objective functions in consideration conflicting. Hence, a pareto plot with various possible optimum cases is generated.

\section{Results and Discussion}

A few of the CFD simulation results are shown in Figures 8, 9 and 10, with their legend represented in Figure 11. These correspond to temperature contours of air with the varying location of chilled beams. The summer extremity temperature of Boston was considered, with the outdoor temperature being $35^{\circ} \mathrm{C}$. These results are plotted on a plane cutting across the middle of the room, perpendicular to the plane of the external window.

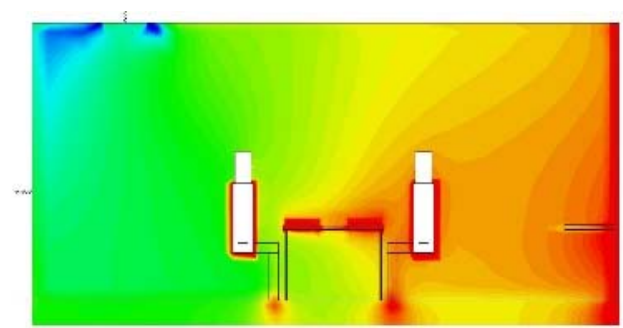

Figure 8: Temperature distribution of air on a plane cutting through the middle of the room, with $C B$ located away from the external window-external temp of $35^{\circ} \mathrm{C}$

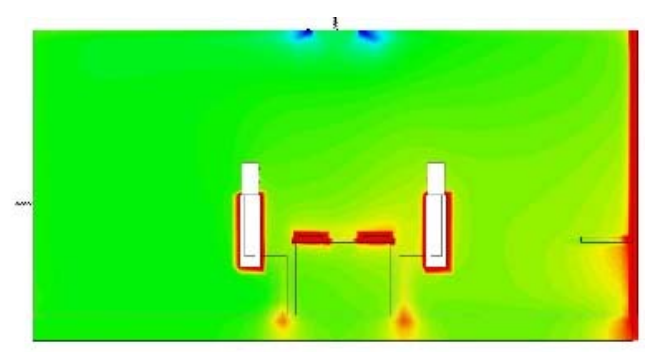

Figure 9: Temperature distribution of air on a plane cutting through the middle of the room, with CB located in the middle of the room-external temp of $35^{\circ} \mathrm{C}$

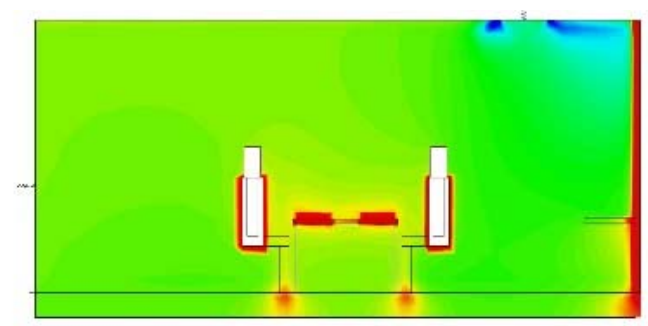

Figure 10: Temperature distribution of air on a plane cutting through the middle of the room, with $C B$ located next to the external window- external temp of $35^{\circ} \mathrm{C}$ 


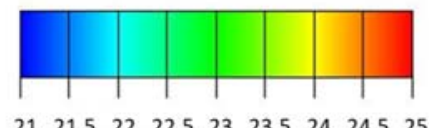

$\begin{array}{lllllllll}21 & 21.5 & 22 & 22.5 & 23 & 23.5 & 24 & 24.5 & 25\end{array}$

Figure 11: Temperature legend for the contour plots in Figures 8, 9 and 10.

Table 1 shows the sample points generated from LHS and their corresponding CFD simulation results of the objective function values. This data was provided as input to the Kriging algorithm to train, test and generate a model that was utilized by GA to optimize the PMV and time values simultaneously. The optimal Pareto front is shown in Figure 12.

Objective 1: Mean PMV

Objective 2: Operational time of air-conditioning (min)

Table 1: Variable and objective function values sampled using Latin Hyper-Cube Sampling

\begin{tabular}{|c|c|c|c|}
\hline $\mathbf{x} / \mathbf{L}$ & $\mathbf{z} / \mathbf{W}$ & $\mathbf{P M V}$ _mean & time (min) \\
\hline 0.333 & 0.1921 & 1.43 & 11.6 \\
\hline 0.2565 & 0.6721 & 1.2 & 8.2 \\
\hline 0.4744 & 0.8537 & 0.94 & 12.8 \\
\hline 0.0046 & 0.0801 & 1.92 & 6.8 \\
\hline 0.1077 & 0.5099 & 1.71 & 7.9 \\
\hline 0.9938 & 0.792 & 0.75 & 29.4 \\
\hline 0.8972 & 0.3552 & 0.64 & 28.9 \\
\hline 0.5403 & 0.2886 & 0.87 & 13.5 \\
\hline 0.7098 & 0.4186 & 0.65 & 18.5 \\
\hline 0.663 & 0.9852 & 0.68 & 15.8 \\
\hline
\end{tabular}

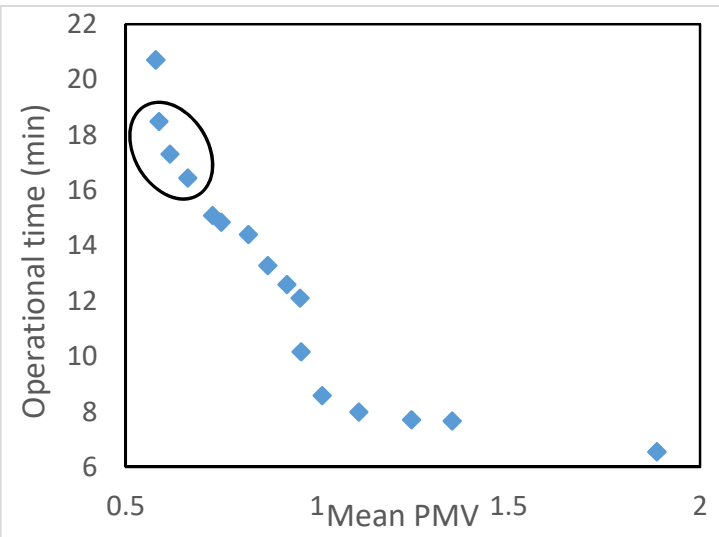

Figure 112: Pareto front showing the optimal objective function values, obtained using Genetic Algorithms
The ideal PMV to be maintained for comfort conditions in the room is around $0.5-0.75$. Looking at these particular values from the Pareto front, we have the optimum set of locations for the chilled beam placement. This optimal set of objective function values are shown in Table 2, along with their corresponding variable values.

Table 2: Optimal solution of variables with their corresponding objective function values, as simulated using Kriging-Genetic Algorithms

\begin{tabular}{|c|c|c|c|c|}
\hline Sl. No & PMV_mean & Time & x/L & z/W \\
\hline 1 & 0.58 & 20.716 & 0.79 & 0.80 \\
\hline 2 & 0.58 & 20.714 & 0.79 & 0.40 \\
\hline 3 & 0.59 & 18.486 & 0.75 & 0.44 \\
\hline 4 & 0.62 & 17.311 & 0.71 & 0.84 \\
\hline 5 & 0.66 & 16.446 & 0.67 & 0.67 \\
\hline 6 & 0.73 & 15.093 & 0.62 & 0.40 \\
\hline 7 & 0.75 & 14.851 & 0.61 & 0.77 \\
\hline
\end{tabular}

The best choices among the optimal solution set correspond to the PMV values lower than 0.6 and operational time around 18-20 minutes (S1. Nos 1,2 and 3 ). They all consistently suggest an $\mathrm{x} / \mathrm{L}$ ratio of around $0.75-0.8$. This $\mathrm{x} / \mathrm{L}$ ratio indicates that the optimal location for a chilled beam is almost half-way between the centre of the room and the external window. If the chilled beams are located too close to the external window or any other solid wall, the air flow from the outlet of the CB might deflect off of the wall and return directly to its inlet, without contributing to cooling of the room air. Hence, a chilled beam is effective when located adequately away from any solid structures. Additionally, chilled beams shouldn't be located directly over occupancy areas, as they result in a draft and reduce the comfort in those spaces. Having the chilled beam away from the external window results in higher horizontal temperature stratification as shown in Figure 8, leading to thermal discomfort for people closer to the external window, especially during the peak season. The above phenomena justify the optimal value of $\mathrm{x} / \mathrm{L}$ $=0.75-0.8$. However, the results suggest two optimal points for $\mathrm{z} / \mathrm{W}$ value- one around 0.8 and another around 0.4. One of the possible influential factors for this optimal value is the location of the door- the only source of air discharge from the room. Oher influential 
factor determining the optimal $\mathrm{z} / \mathrm{W}$ value is the location of the heat sources. Assuming that most of the heat sources are concentrated in the centre of the room, the ideal position to have the chilled beam is slightly away from the centre on either side, depending on the location of the door. A $\mathrm{z} / \mathrm{W}$ value of 0.5-0.6 would place the chilled beam right over the heat sources and would result in a potential draft underneath it.

To test the efficacy of the Kriging model, a CFD simulation was run with the above mentioned optimum results (optimum result no 1). The simulation results indicated a PMV value of 0.57 and a transient operational time of $20.2 \mathrm{~min}$. This is within 5\% error as compared to the predicted Kriging-GA optimum results.

\section{Conclusion}

A detailed CFD analysis of a chilled beam system in a room containing an external window was performed. Experiments were conducted in a test room for validating the CFD results. Cases of varying position of the chilled beam were simulated and data points collected to provide as inputs to the Kriging model. This surrogate model was later optimized using Genetic Algorithms. The results indicated that the ideal position of the chilled beam would be around the three-fourths mark $(\mathrm{x} / \mathrm{L}=0.75-0.8)$ in the room towards the external window, with some slight offset $(\mathrm{z} / \mathrm{W}=0.4$ or 0.8$)$ to the centre in its perpendicular direction. This is also backed by the understanding that chilled beams would be less efficient when placed very close to solid objects and right above heat sources. This would not only work for rooms with external windows, but to all mid-sized rooms in general. Rooms with very large occupancy volume might need multiple chilled beams to be installed, which would require a separate analysis. A much more comprehensive energy analysis using simulation software like Energy Plus in the future would give a better understanding.

\section{Acknowledgement}

The authors would like to acknowledge the support and resources provided by Schneider Electric for conducting the research. This research was also sponsored by the MIT-HKUST Consortium.

\section{Nomenclature}

c Specific heat capacity

e Emissivity

F Area parameter

Fcl Clothing insulation parameter

g Acceleration due to gravity
H Metabolic Heat Production

Hc Convective heat transfer coefficient

$\mathrm{k}$ thermal conductivity

P Pressure

$\mathrm{Pa} \quad$ Vapour Pressure of Water Vapour

q Heat or Energy

T Temperature

Ta Air temperature

Tcl Clothing surface temperature

$\operatorname{Tr} \quad$ Mean radiant temperature

U Velocity Vector

$\rho \quad$ Density

$\sigma \quad$ Stephan-Boltzmann constant

$\mu \quad$ Dynamic viscosity

\section{References}

Alexander, D., \& O'Rourke, M. (2008). Design considerations for active chilled beams. ASHRAE Journal, 50(9), 50-58.

Fanger, P. O. (1970). Thermal comfort. Analysis and applications in environmental engineering. Thermal comfort. Analysis and applications in environmental engineering.

Ken Loudermilk, P. E. (2009). Chilled Beams Thermal Comfort FOR.

Kim, J., Tzempelikos, A., Horton, W. T., \& Braun, J. E. (2018). Experimental investigation and datadriven regression models for performance characterization of single and multiple passive chilled beam systems. Energy and Buildings, 158, 1736-1750.

Koskela, H., Häggblom, H., Kosonen, R., \& Ruponen, M. (2012). Flow pattern and thermal comfort in office environment with active chilled beams. $H V A C \& R$ Research, 18(4), 723-736.

Lophaven, S. N., Nielsen, H. B., \& Søndergaard, J. (2002). DACE: a Matlab kriging toolbox (Vol. 2). IMM, Informatics and Mathematical Modelling, The Technical University of Denmark.

Muller, D., Gores, I., \& Zielinski, R. (2004, September). Impact of the thermal load on the room airflow pattern. In RoomVent 2004 9th International Conference on Air Distribution in Rooms (pp. 5-8).

Nielsen, P. V., Allard, F., Awbi, H. B., Davidson, L., \& Schälin, A. (2007). Computational Fluid Dynamics in Ventilation Design REHVA Guidebook No 10.

Wu, B., Cai, W., Chen, H., \& Ji, K. (2018). Experimental investigation on airflow pattern for 
active chilled beam system. Energy and Buildings, 166, 438-449.

Zbořil, V., Melikov, A., Yordanova, B., Bozhkov, L., \& Kosonen, R. (2007). Airflow distribution in rooms with active chilled beams. In Proceedings of the 10th International Conference on Air Distribution in Rooms-Roomvent 2007 (pp. 1-7). 